\title{
Partial Response by Computed Tomography
}

National Cancer Institute

\section{Source}

National Cancer Institute. Partial Response by Computed Tomography. NCI Thesaurus.

Code C123610.

A decrease in the size and extent of tissue involvement by cancer, based on CT scan and criteria-defined assessments. 